\title{
Descripción de dos casos teratológicos en Leptoglossus concaviusculus Berg, 1892 (Heteroptera: Coreidae) de la Provincia de Río Negro, Argentina
}

\author{
Description of two teratological cases in Leptoglossus concaviusculus Berg, 1892 \\ (Heteroptera: Coreidae) from Río Negro Province, Argentina
}

Javiera R. Rocca ${ }^{1} \&$ Eduardo I. Faúndez ${ }^{2,3}$

\section{Resumen}

Leptoglossus concaviusculus Berg, 1892, es una especie de Coreidae que se distribuye en Argentina, Brasil, Paraguay y Uruguay. En la presente contribución se describen dos casos teratológicos en L. concaviusculus. El primero asociado a un individuo macho, trata de una oligomería unilateral simple y el segundo asociado a un individuo hembra, trata sobre una atrofia. Se discuten las posibles causas asociadas a los daños, y se reporta por primera vez parasitismo por parte de Tachinidae (Diptera) en esta especie.

\section{Palabras clave:}

Teratosis, Hemiptera, Coreinae,

Tachinidae, Patagonia.

\begin{abstract}
Leptoglossus concaviusculus Berg, 1892 is a leaf-footed bug distributed in Argentina, Brazil, Paraguay, and Uruguay. In this contribution, two teratological cases in $L$. concaviusculus are described. The first one is a simple oligomery in a male specimen, and the second belongs to a case of atrophy on a female specimen. Possible causes of the teratosis are discussed. It is also provided the first record of parasitism by Tachinidae (Diptera) on this species.
\end{abstract}

\section{Key words:}

Teratosis, Hemiptera, Coreinae, Tachinidae, Patagonia.

\section{INTRODUCCIÓN}

La familia Coreidae se compone de alrededor de 2550 especies distribuidas en unos 436 géneros (Faúndez, 2015). Leptoglossus Guérin-Méneville, 1831 es uno de los géneros más diversos y complejos de la tribu Anisoscelini en el hemisferio occidental (Brailovsly, 2014) y se encuentra compuesto en la actualidad por 62 especies (Faúndez \& Carvajal, 2016); varias de estas consideradas de importancia económica (Faúndez et al. 2017, Faúndez \& Rocca, 2017) y en algunos casos altamente invasivas (Faúndez \& Rocca, 2017). Leptoglossus concaviusculus Berg, 1892, anteriormente conocida como subespecie de Leptoglossus chilensis (Spinola, 1852); es en la actualidad una especie (Faúndez \& Carvajal, 2016) que se distribuye por Argentina, Brasil, Paraguay y Uruguay, y se diferencia de las especies del grupo de L. chilensis principalmente por el gran tamaño de las dilataciones foliares de la metatibia, la ausencia de manchas discoidales en la zona posterior del pronoto, ángulos humerales

1 Instituto de Biología, Facultad de Ciencias, Pontificia Universidad Católica de Valparaíso, Av. Universidad 330, Placilla (Curauma), Región de Valparaíso, Chile

2 Entomology Department, School of

Natural Resource Sciences, North Dakota State University, Dept. 7650, P.O. Box 6050; Fargo, ND, USA.

$\measuredangle$ ed.faundez@gmail.com

3 Instituto de la Patagonia, Universidad de Magallanes, Av. Bulnes, 01855, Punta Arenas, Chile. 
ensanchados, cabeza elongada y pigóforo ovoidal de lados convexos (Faúndez \& Carvajal, 2016). Esta especie ha sido considerada de una moderada importancia económica, y también, se le ha registrado picando de manera adventicia a un ser humano (Faúndez \& Carvajal, 2011).

La teratología comprende el estudio de las malformaciones y anormalidades (Carvajal \& Faúndez, 2016). El objetivo de esta contribución será describir dos casos teratológicos en $L$. concaviusculus.

\section{MATERIALES Y MÉTODOS}

Los individuos descritos fueron obtenidos en una serie de 11 ejemplares colectados en la Provincia de Río Negro, Patagonia Argentina; y se encuentran depositados en la colección de referencia de Heterópteros del Centro de Estudios en Biodiversidad en Osorno, Chile. Las fotografías fueron tomadas con una cámara digital Ricoh WG-4 con una resolución de $16 \mathrm{mp}$; adaptada a un microscopio estereoscópico. La descripción teratológica se hizo siguiendo la terminología y clasificación de Štusak \& Stehlik (1978).

\section{RESULTADOS}

Caso 1. Oligomería unilateral en antena izquierda (Tabla I, Figs. 1, 2)

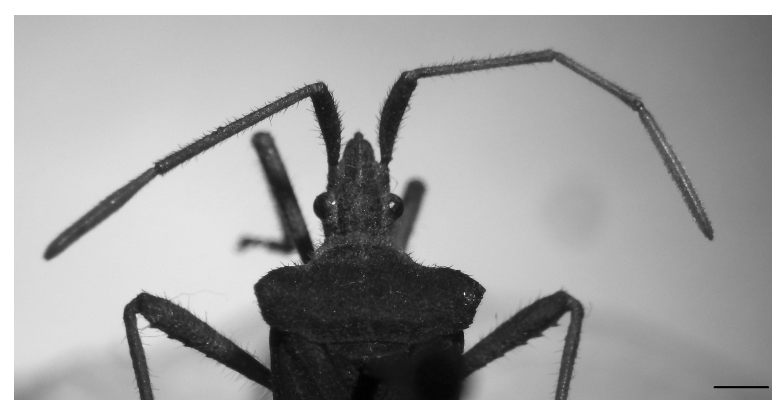

Fig. 1. Oligomería unilateral izquierda en L. concaviusculus. Escala $=1 \mathrm{~mm}$.

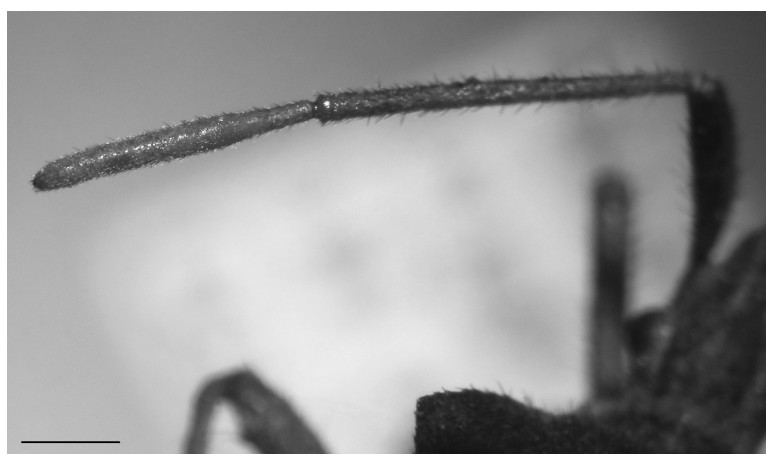

Fig. 2. Oligomería unilateral izquierda en $L$. concaviusculus, detalle en últimos antenómeros. La Escala $=1 \mathrm{~mm}$.

Material examinado: Argentina, Río Negro, E de General Roca, 3-II-2017, J. Muñoz leg. $10^{7}$.

El individuo evidencia la falta del último artejo antenal izquierdo (Fig.1). Se observa según mediciones que los artejos II y III de la antena malformada son cerca de un milímetro más largos que sus pares de la antena derecha normal (Tabla I), además que el mismo artejo III teratológico presenta en sus dos tercios apicales un engrosamiento y pilosidad de las misma características morfológicas del artejo IV de la antena derecha (Fig. 2). Finalmente, la articulación de la estructura malformada aparenta ser funcional, por lo que este caso se trataría de una oligomería simple sin anartrogénesis.

\section{Caso 2. Atrofia en antena izquierda (Tabla II, Figs. 3, 4)}

Material examinado: Argentina, Río Negro, E de General Roca, 3-II-2017, J. Muñoz leg. 19.

El individuo posee la totalidad de artejos de ambas antenas, sin embargo, presenta una disminución en la longitud de los artejos II, III y IV de la antena izquierda (Tabla II; Fig. 3). La antena malformada presenta pilosidad en su totalidad de las mismas características que su par derecho, y sus articulaciones parecen funcionales (Fig. 4).

Tabla 1. Medidas en milímetros de las antenas del ejemplar del caso 1.

\begin{tabular}{lccccc}
\hline Antenómero & I & II & III & IV & Total \\
\hline Antena izquierda & 1,67 & 3,55 & 2,76 & - & 7,98 \\
Antena derecha & 1,67 & 2,66 & 1,87 & 2,76 & 8,96
\end{tabular}


Tabla 2. Medidas en milímetros de las antenas del ejemplar del caso 2.

\begin{tabular}{lccccc}
\hline Antenómero & I & II & III & IV & Total \\
\hline Antena derecha & 1,74 & 2,89 & 1,96 & 1,92 & 8,51 \\
Antena izquierda & 1,74 & 2,71 & 1,63 & 2,25 & 8,33 \\
\hline
\end{tabular}

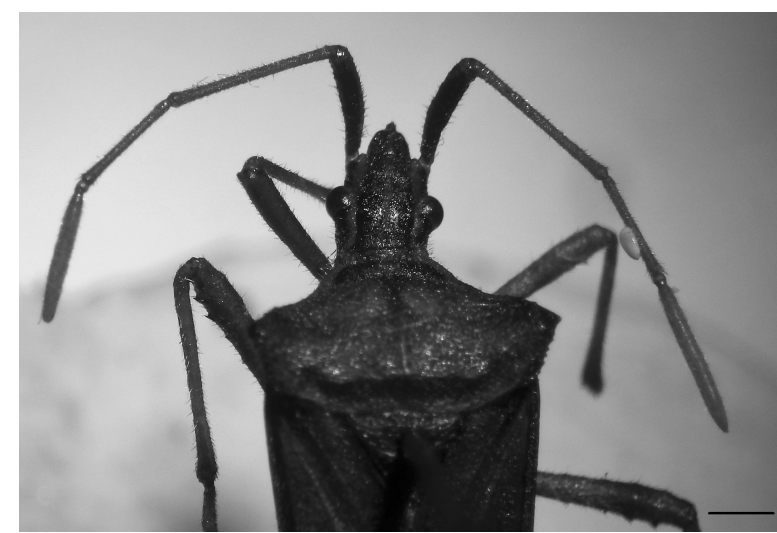

Fig. 3. Atrofia antenal izquierda en L. concaviusculus. Escala $=1 \mathrm{~mm}$.

Cabe destacar que el ejemplar presenta un huevo de Tachinidae (Diptera) en la antena izquierda.

\section{DISCUSIÓN Y CONCLUSIONES}

El grado de regeneración de una lesión antenal depende en gran medida del estado ninfal en que se haya producido el hecho durante los estados juveniles. Mientras más prematuro haya sido el instar en que se encontraba el individuo además de la gravedad de la lesión es como esta se regenerará en el tiempo (Stusak \& Stehlik, 1978). En el caso número 1, creemos que la lesión que terminó con la pérdida total de uno de sus artejos se produjo en un instar temprano, ya que el proceso de recuperación y regeneración terminó por dar al artejo III las estructuras necesarias para cumplir las funciones que cumpliría el artejo IV. Esto se observa al comparar la morfología del artejo III de la antena izquierda con el artejo IV de la antena derecha. El cambio en la pilosidad y el engrosamiento son típicas respuestas regeneratorias en heterópteros Pentatomomorfos (Stusak \& Stehlik, 1978).

Las atrofias pueden deberse a lesiones pequeñas ya sean heridas o enfermedades que degeneren en un estrechamiento de los segmentos antenales o de la antena en su totalidad. En el

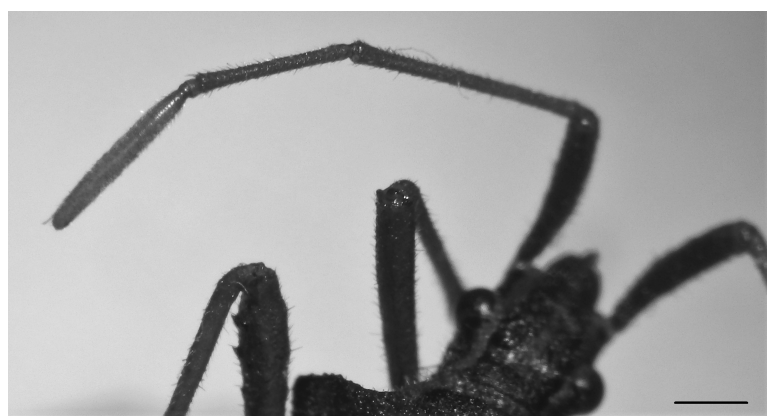

Fig. 4. Atrofia antenal izquieda en $L$. concaviusculus, detalle antena malformada. Escala $=1 \mathrm{~mm}$.

caso número 2, creemos poder asociar este estrechamiento del largo de los segmentos a la presencia parasitoide de un huevo de Tachinidae en la otra antena del individuo. Tachinidae es una familia de dipteros compuesta por alrededor de 10.000 especies (Gammelmo \& Arve, 2007) y se destacan por sus características parasitoides en otros artrópodos, principalmente insectos fitófagos (Hawkins, 1994). En Hemiptera, se han descrito casos donde las moscas de esta familia han parasitado algunas especies de las familias Pentatomidae, Coreidae y Lygaeidae (Arnaud, 1978). De este modo, la antena malformada podría haber sido parasitada anteriormente en algún estado ninfal, sin perder un segmento, pero afectando su nivel de desarrollo, resultando en la reducción de tamaño. En este sentido, es posible que la lesión ocurrida en el caso número 1 , también pueda deberse a algún ataque de Tachinidae, ya que otros tres ejemplares en la serie colectada presentan huevos en otras partes del cuerpo (i.e. pronoto y vértex).

Las anomalías antenales son relativamente comunes en heteroptera (Stusak \& Stehlik, 1978); sin embargo, estos son los primeros casos descritos en coreidos sudamericanos. A futuro queda investigar con mayor profundidad la interacción entre los Tachinidae y L. concaviusculus, además de indagar las consecuencias que pueden traer 
estas interacciones en otros heterópteros, especialmente aquellos grupos que poseen mayor cantidad de casos reportados de teratologías.

\section{LITERATURA CITADA}

Arnaud, P. (1978). Host parasite catalog of North American Tachinidae (Diptera). Washington D. C. United States of America. United States Department of Agriculture, 860pp.

Berg, C. (1892). Nova Hemiptera Argentinae et Uruguayensis. Anales de la Sociedad Científica Argentina, 33, 65-72.

Brailovsky, H. (2014). Illustrated key for identification of the species included in the genus Leptoglossus (Hemiptera: Heteroptera: Coreidae: Coreinae: Anisoscelini), and descriptions of five new species and new synonyms. Zootaxa, 3794(1), 143-178.

Carvajal, M. A., \& Faúndez, E. I. (2016). A teratological case in the family Idiostolidae (Hemiptera: Heteroptera: Idiostoloidea). Anales del Instituto de la Patagonia 44 (1), 43-46.

Faúndez E. I. (2015). Synopsis of the genus Eldarca Signoret, 1864 (Hemiptera: Heteroptera: Coreidae). Zootaxa, 3915(2), 287-294.

Faúndez, E. I., \& Carvajal, M. A. (2011). Primer registro de una picadura de Leptoglossus chilensis (Spinola, 1852) (Hemiptera: Heteroptera: Coreidae) en un ser humano.
Boletín de Biodiversidad de Chile, 6, 2225.

Faúndez E. I. \& Carvajal M. A. (2016). Sobre la relación entre Leptoglossus chilensis (Spinola, 1852) y Leptoglossus concaviusculus Berg, 1892 stat. rest. (Heteroptera: Coreidae), con nuevos datos acerca de su morfología. Anales del instituto de la Patagonia, 44(3), 65-70.

Faúndez, E. I., Rocca, J. R. \& Villablanca, J. (2017). Detection of the invasive western conifer seed bug Leptoglossus occidentalis Heidemann, 1910 (Heteroptera: Coreidae: Coreinae) in Chile. Arquivos Entomolóxicos, 17, 317320.

Faúndez, E. I., \& Rocca, J. R. (2017). La chinche de las coníferas occidental, Leptoglossus occidentalis Heidemann (Heteroptera: Coreidae) en Chile; rápida expansión, posibles impactos y desafíos. Revista Chilena de Entomología, 42, 25-27.

Gammelmo, Ø., \& Arve, B. (2007). The tachinid fly Phasia hemiptera (Fabricius, 1794) (Diptera, Tachinidae) in Norway. Norway Journal of Entomology, 54, 51-54.

Hawkins, B. A. (1994). Patterns and process in host-parasitoid interactions. Cambridge University Press, Cambridge, Mass. 190pp.

Stusak, J. M. \& Sthelik, J. L. (1978). Second contribution to the teratology of Tingidae (Heteroptera) Antennal anomalies. Acta Musei Moraviae, Scientiae biologicae, 63, 89-105. 